Widefield Science and Technology for the SKA

SKADS CONFERENCE 2009

S.A. Torchinsky, A. van Ardenne, T. van den Brink-Havinga, A.J.J. van Es, A.J. Faulkner (eds.)

4-6 November 2009, Château de Limelette, Belgium

\title{
FIDA $^{3}$ : a novel active array for the mid-SKA
}

\author{
O. García-Pérez ${ }^{1,2}$, J. A. López-Fernández ${ }^{1}$, D. Segovia-Vargas ${ }^{2}$, L. E. García-Muñoz ${ }^{2}$, V. González-Posadas ${ }^{3}$, \\ J. L. Vázquez-Roy ${ }^{2}$, J. M. Serna-Puente ${ }^{1}$, E. Lera-Acedo ${ }^{1,2}$, T. Finn ${ }^{1}$, P. Colomer ${ }^{1}$, and R. Bachiller ${ }^{1}$ \\ 1 Observatorio Astronómico Nacional, Instituto Geográfico Nacional (IGN), Yebes, Spain \\ e-mail: oscar.perez@oan.es * \\ ${ }^{2}$ Department of Teoría de la Señal y Comunicaciones, Universidad Carlos III de Madrid (UC3M), Leganés, Spain \\ 3 Department of Ingeniería Audiovisual y Comunicaciones, Universidad Politécnica de Madrid (UPM), Madrid, Spain
}

\begin{abstract}
This paper presents an active array demonstrator for the mid-frequency range (i.e.: from $300 \mathrm{MHz}$ to $1000 \mathrm{MHz}$ ) of the Square Kilometre Array (SKA). We describe the design process of FIDA ${ }^{3}$ (FG-IGN Differential Active Antenna Array), which is the FG-IGN contribution for the task DS4-T4 of the SKADS project. The main advantages provided by this design include the use of a dielectric-free structure, and the use of differential amplifiers directly connected to the feeding lines of the antennas. The array has been optimized in order to exhibit well active impedance matching properties in the band of interest. On the other hand, the differential low noise amplifiers are designed in order to obtain a very low noise temperature for such antenna impedance. A $1 \mathrm{~m}^{2}$ array tile has been manufactured, and both the antenna impedance and the amplifier noise performance have been measured. Thus, the radiating structure provides scanning capabilities up to \pm 45 deg with acceptable impedance matching. With respect to the amplifiers, the measured noise temperature is better than $40 \mathrm{~K}$.
\end{abstract}

\section{Introduction}

The Square Kilometre Array (SKA) will be a next generation radio-telescope with more than $1 \mathrm{~km}^{2}$ collecting area (Dewdney et al. 2009). This instrument is intended to operate in the frequency range from $70 \mathrm{MHz}$ to $10 \mathrm{GHz}$, and it will be much more sensitive than current radio-telescopes. In this context, different antenna technologies are proposed in order to cover the full bandwidth: arrays of dipole-like antennas in random sparse configuration for the lowest frequency band (i.e.: from $70 \mathrm{MHz}$ to $450 \mathrm{MHz}$ ), aperture arrays of tapered slot antennas (TSA) for the mid-frequency range (i.e.: from $300 \mathrm{MHz}$ to $1000 \mathrm{MHz}$ ), and small dish reflectors at high frequencies (i.e.: from $1 \mathrm{GHz}$ to $10 \mathrm{GHz}$ ). This paper focuses in the design of an active array prototype, whose technology can be a potential candidate for the mid-frequency range of the SKA. The proposed solution provides different advantages. The use of dielectric-free antennas allows saving costs and avoids the potential losses of a substrate board. Furthermore, the use of differential low noise amplifiers (LNA) directly connected to the antennas avoids the losses and bandwidth restrictions provided by passive baluns. However, many limitations may appear in both the antenna and LNA designs, precisely due to their differential topology. Firstly, the radiating structure may propagate common-mode currents that can cause scan impedance anomalies. On the other hand, the design and characterization of differential amplifiers is a non trivial task that needs the use of some specific techniques. Next sections show how all the cited problems are overcome.

\footnotetext{
^ This work was supported by the European Commission Framework Program 6, Project SKADS, Square Kilometre Array Design Studies (SKADS), contract no 011938.
}

\section{Radiating structure}

The mid-frequency range of the SKA is projected to be covered by a dense array of millions of cheap antennas. These arrays should be dual-polarized, and should be able to scan up to $\pm 45 \mathrm{deg}$. The benefits of using TSAs instead of other ultrawideband elements in this type of dense array configurations with strong mutual coupling effects has been already reported in the literature by some previous works (van Ardenne et al. 2003). Thus, it is possible to create a grid of TSAs electrically interconnected, and obtaining a good reflection coefficient in a frequency band as large as 3:1. Fig. 1 shows different types of TSA elements. The first three types correspond to classical Vivaldi antennas that are fed by means of a transmission line acting as a balun. The other two types are bunny-ear antennas that can fed in differential mode. Another classification can be done attending to the use of a dielectric layer in the antenna structure. This paper makes use of the bunny-ear antenna presented in Fig. 1.e, which presents several interesting advantages for this application. Firstly, the potential losses introduced by a substrate layer are avoided, since the antenna is formed by a single metallic layer. Additionally, a differential LNA can be attached to the antenna, avoiding the losses caused by a passive balancing feeding board, which is critical for low noise performance. The antenna element and the array configuration are shown in Fig. 2.

The design and optimization process of this antenna array has been presented in (Lera et al. 2010). A parametric study over the main geometric parameters of the antenna has been carried out, in order to obtain a low VSWR with respect to $150 \Omega$ in differential mode. The array should operate in the $300 \mathrm{MHz}$ to $1000 \mathrm{MHz}$ bandwidth with a scanning range up to $\pm 45 \mathrm{deg}$. This simulation was done by using the commercial full-wave simulator HFSS from Ansoft, assuming 
an infinite-by-infinite dual-polarized array, and calculating the corresponding active reflection coefficient (Pozar 2009) of the antenna elements. One of the main contributions in (Lera et al. 2010) is the analysis and dicussion about a new type of scan anomaly that was not previously reported in the literature. This anomaly is related with the propagation of even-mode currents in the antenna array. As shown in Fig. 3.a, the conventional performance is given by the propagation of odd-mode currents between the differential feeding lines of the antennas. However, it has been demonstrated that this differential structure over a GND plane can also propagate odd-mode currents at some frequencies and scanning angles (see Fig. 3.b). As shown in Fig. 4, the effect is the appearance of scan blindness effects in the frequency response of the array when scanning at angles different to broadside. In practice, these anomalies may also appear at broadside due to the unavoidable asymmetries in the manufactured array. The proposed solution in Lera et al. (2010) consists of connecting two resistors between the feeding lines of the antennas and the GND plane, which are used to dissipate the common-mode currents in the antennas (see Fig. 3.c). There are several trade-offs that must be considered when deciding the value of such resistors. These resistors should be low enough in order to guide the odd-mode currents to the GND plane. However, lower values of the resistor will increase the noise temperature of the system, as it is plotted in Fig. 3.d. Finally, a value of the resistor close to $3 \mathrm{k} \Omega$ has been chosen as the most adequate in order to avoid the resonances and keep a low VSWR with the minimum cost in terms of noise. In this case, this noise contribution is lower than $10 \mathrm{~K}$ in the band of interest. The active reflection coefficient of the optimized antenna array is shown in Fig. 5. The VSWR is lower than 2.5:1 in the band of interest for scanning angles from broadside to $45 \mathrm{deg}$.

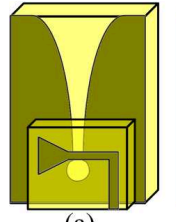

(a)

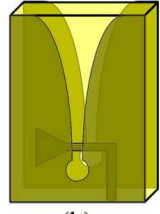

(b)

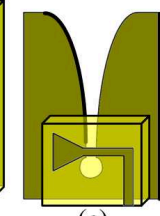

(c)

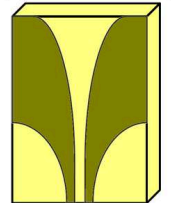

(d)

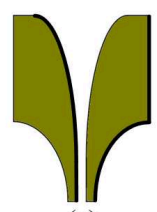

(e)
Fig. 1: Different types of tapered slot antenna elements: (a) printed on a substrate with microstrip feed; (b) printed on a dielectric substrate with stripline feed; (c) one layer of metal with microstrip feed; (d) printed on a substrate with differential feed; (e) one layer of metal with differential feed.

A photograph of the manufactured array prototype is shown in Fig. 6. It consists of 64 bunny-ear antenna elements placed in a grid in order to provide dual polarization. The antenna structure, the GND plane, and the metallic box under the GND

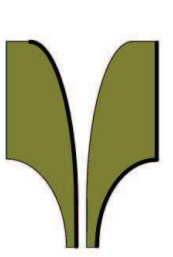

(a)

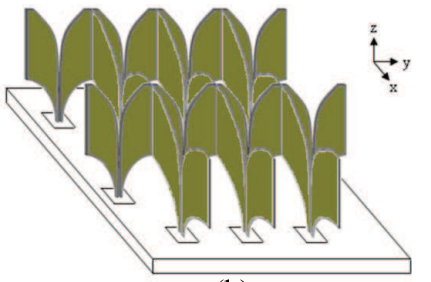

(b)
Fig. 2: Bunny-ear antenna element (a) and array configuration (b).
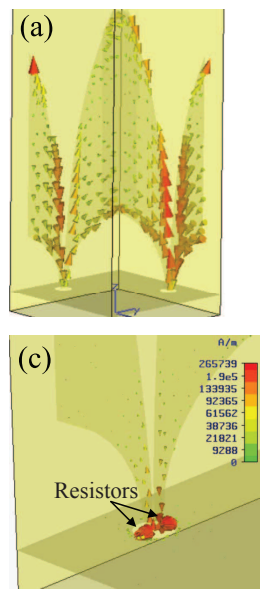
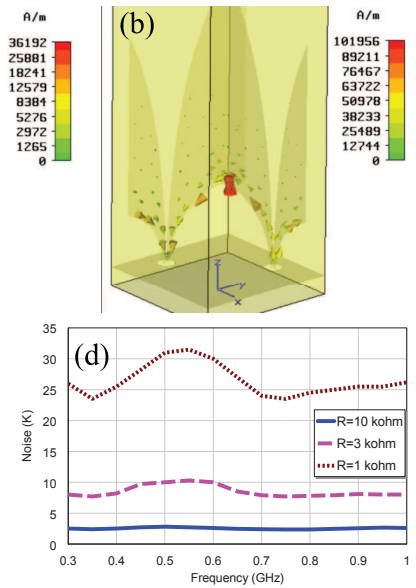

Fig. 3: Surface currents in the antenna at $500 \mathrm{MHz}$ (a) and at the anomaly frequency (b); proposed solution for the anomalies (c); and extra noise contribution for different values of the resistor (d).

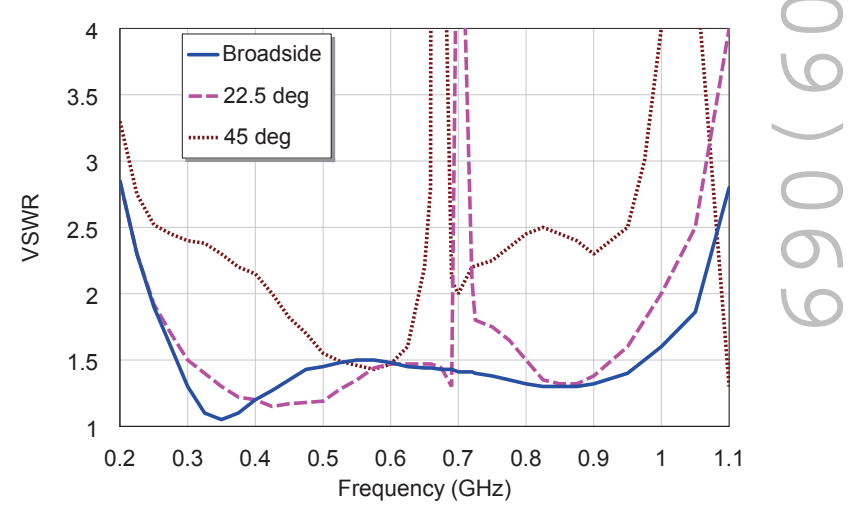

Fig. 4: Simulated VSWR for different scanning angles in the E-plane before correcting the scan anomalies.

plane have been built in aluminium. The S-parameter matrix of the array has been measured by using passive baluns in order to convert from differential to single-ended mode. By means of this S-parameter matrix, which contains the information of the mutual coupling coefficients in the array, it can be obtained the active reflection coefficient of any antenna element (Pozar 2009). Fig. 7 shows the calculated active reflection coefficient of the centre element of the array, for different scanning angles. It can be seen how the active reflection coefficient is better than $-6 \mathrm{~dB}$ in the band of interest. Fig. 8 shows the measured active 


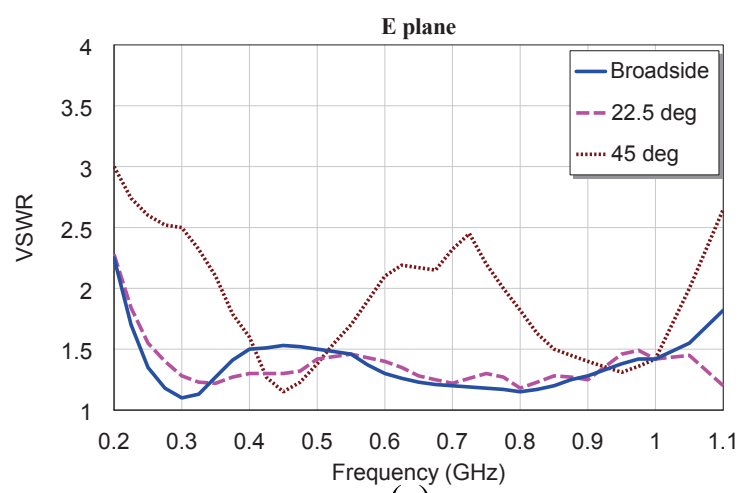

(a)

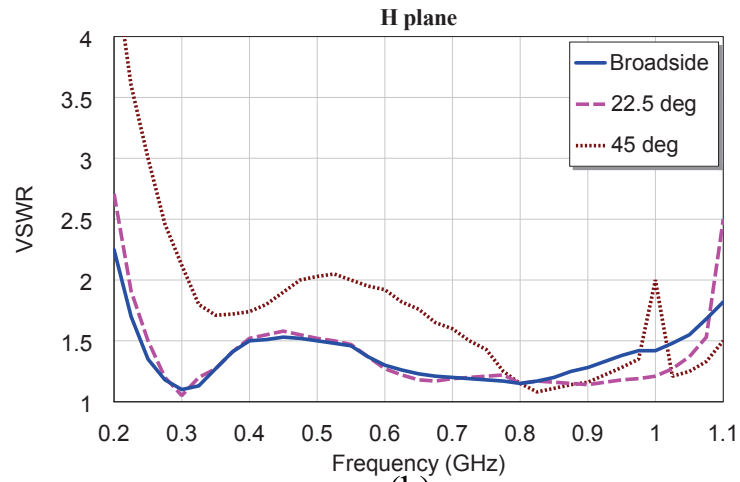

(b)

Fig. 5: Simulated VSWR for different scanning angles in the E-plane (a) and H-plane (b).

reflection coefficient at $800 \mathrm{MHz}$ of the 32 antenna elements corresponding to one of the two polarizations of the array. As it was expected, the centre elements show better matching performance than the edge elements. In any case, the reflection coefficient of most of the elements is lower than $-5 \mathrm{~dB}$ in the band of interest, which is acceptable for this application.

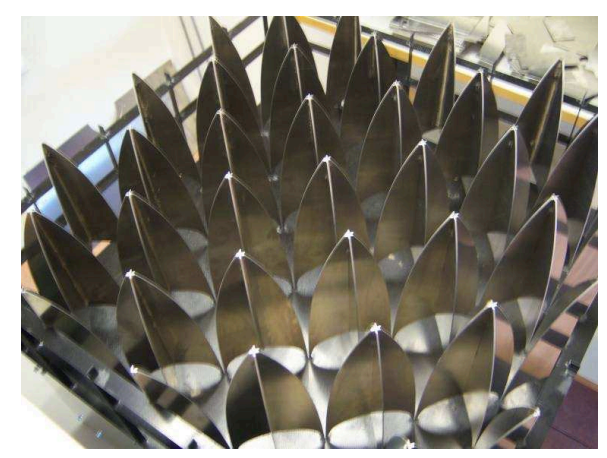

Fig. 6: Photograph of the manufactured array.

\section{Differential amplifiers}

As exposed before, one of the main advantages provided by the bunny-ear antennas is that a differential LNA can be di-

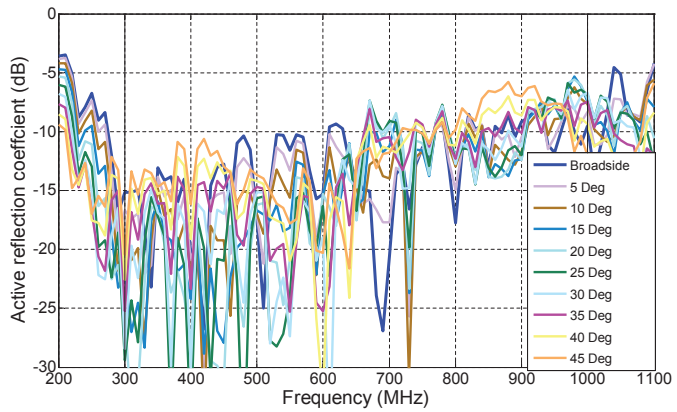

Fig. 7: Measured active reflection coefficient of the centre element of the array for different scannning angles.
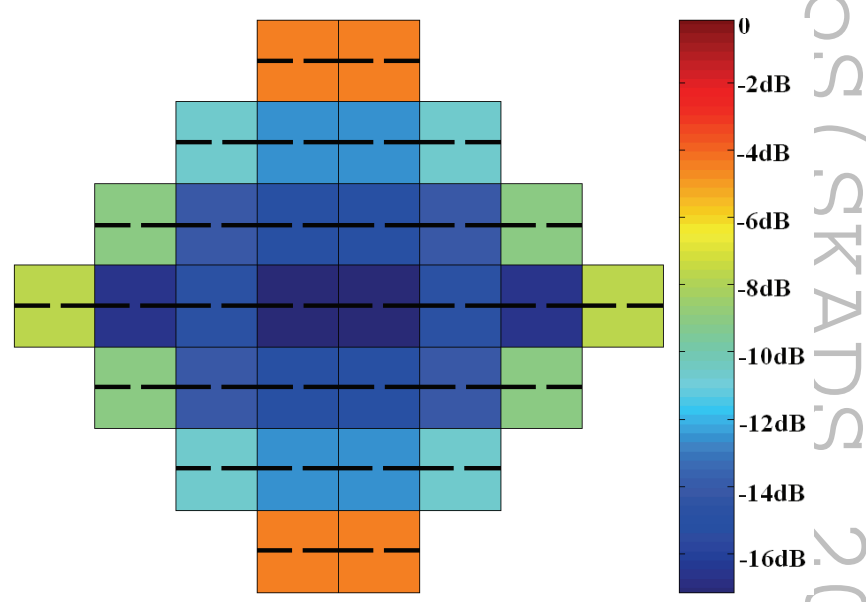

Fig. 8: Measured active reflection coefficient of the 32 elements of the manufactured array tile at $800 \mathrm{MHz}$.

rectly connected to the antenna terminals, avoiding the use of a passive balun. The proposed differential amplifier is shown in Fig. 9. It has been implemented by using lumped components over a microstrip board. The transistor are low noise PHEMTs, model ATF34-143 from Avago Technologies. The inductive de generation technique (Belostotski \& Haslett 2006) has been used in order to obtain a good trade-off between input power matching and low noise performance. The simulations have been carried out by using the Agilent ADS software. The measured S-parameters of the amplifier are shown in Fig. 10 and Fig. 11. They have been obtained by using a multiport network analyzer and assume a source impedance of $150 \Omega$ in differential mode, since this is the average value of the active antenna impedance. The gain is higher than $26 \mathrm{~dB}$ and the input reflection coefficient is better than $-6 \mathrm{~dB}$ in the band of interest. With respect to the noise, the characterization of differential devices is not trivial. The main problem is that conventional noise analyzers provide two single-ended ports, both matched to $50 \Omega$. In this case we need to characterize the amplifier for a differential source impedance of $150 \Omega$. Fortunately, some techniques presented in the literature overcome this problem. The method used in this work consists of connecting a $150 \Omega$ differential load at the input of the amplifier, and measuring the output power when the load is exposed to two different temperatures (Morawietz et al. 2007). Thus, the noise temperature of 
the device under test can be obtained from the ratio between both output powers. In this case, the measured noise temperature is lower than $40 \mathrm{~K}$ in the band of interest, as it can be seen in Fig. 12. Furthermore, good agreement between the measurement and the simulation can be observed.

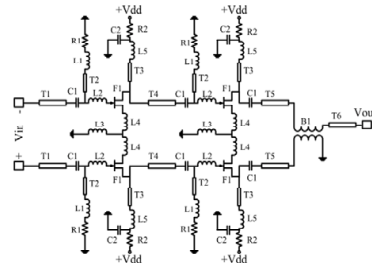

(a)

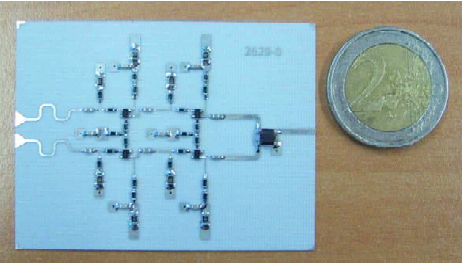

(b)
Fig. 9: Circuit schematic (a) and prototype board (b) of the implemented differential LNA.

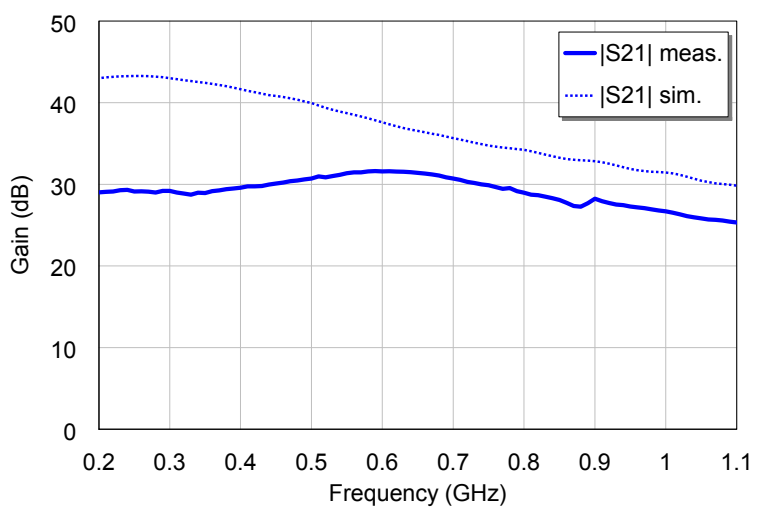

Fig. 10: Measured and simulated gain of the differential LNA.

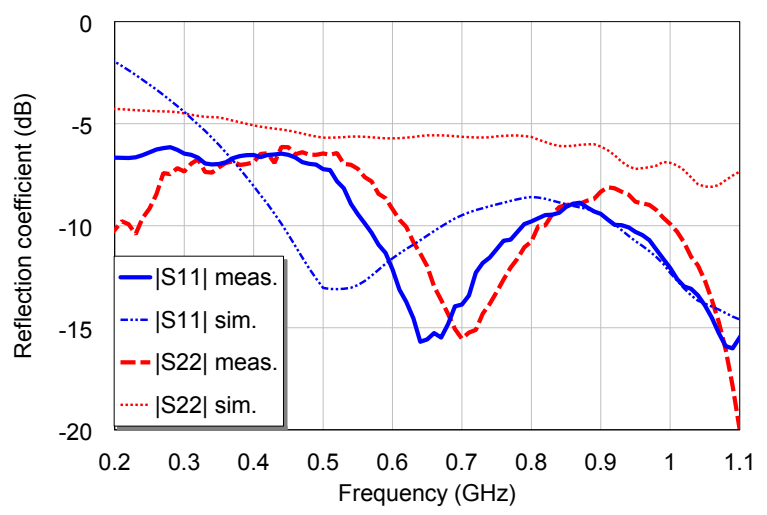

Fig. 11: Measured and simulated reflection coefficients of the differential LNA.

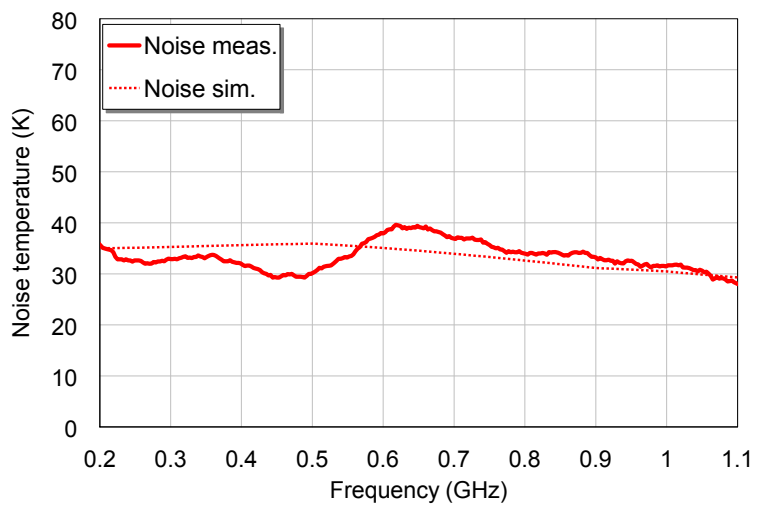

Fig. 12: Measured and simulated noise temperature of the differential LNA.

\section{Receiver characterization}

As it was exposed in section 2 , the active antenna impedance of the array takes into account the mutual coupling effects between the antenna elements. In the case of the present array, all the antennas are assumed to be loaded with $150 \Omega$ during the simulation of the radiating structure (see Fig. 13.a). Thus,

the array is optimized in order to exhibit a low active reflection coefficient with respect to this reference impedance. However, in practice, the array will be loaded by the implemented LNAs that can be not perfectly matched to the reference impedance, and this effect should be considered in order to obtain an accurate characterization of the system (see Fig. 13.b). Fig. 13.c shows the simulated active antenna impedance when the antenna elements are loaded with $150 \Omega$ and when they are loaded with the LNAs presented in section 3. At low frequencies, where the LNA presents a worse input reflection coefficient, the discrepancies in the active antenna impedance are higher. Furthermore, it is precisely at low frequencies where this effect is more pronounced due to higher mutual couplings in the array. This mismatching effect that the LNA causes over the antenna impedance, in turn, causes a variation in the system noise performance. Fig. 14 shows the simulated noise temperature of the LNA for different antenna conditions: $150 \Omega$, the active antenna impedance simulated with ideal LNAs, and the active antenna impedance simulated with the LNA design presented in section 3. Even in the case of the ideal active antenna impedance, which presents matching levels better than $-10 \mathrm{~dB}$, there are discrepancies higher than $10 \mathrm{~K}$ with respect to the performance with a $150 \Omega$ source impedance. In the case of the actual active antenna impedance, these discrepancies are higher, especially at low frequencies at it was expected. Although the previous results correspond to simulations, it is expected to obtain a similar behaviour in measurements, due to the good agreement obtained from the gain and noise characterization of the differential LNA. The measurement of the LNA performance for different source impedance conditions can be obtained from a source-pull characterization, but this procedure presents some difficulties when working with differential devices, since it is necessary to use a differential impedance tuner or a passive input balun (Garcia-Perez et al. 2009). 
(a)
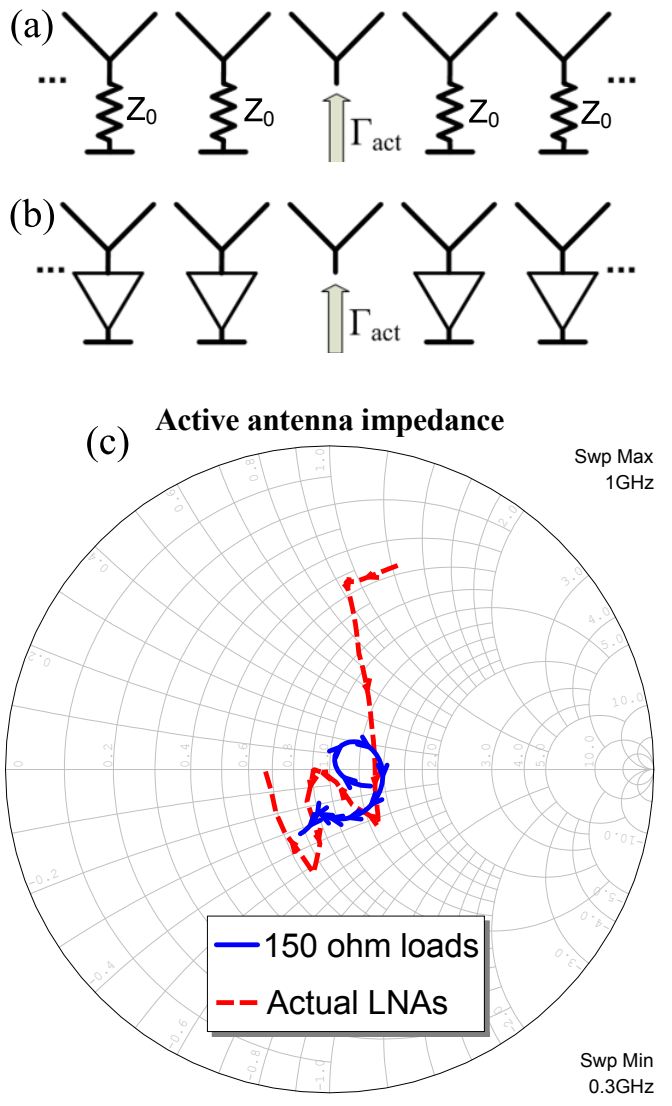

Fig. 13: Characterization of the array loading with ideal loads (a) and with the LNAs (b), and simulated active antenna impedance at broadside obtained in both cases (c).

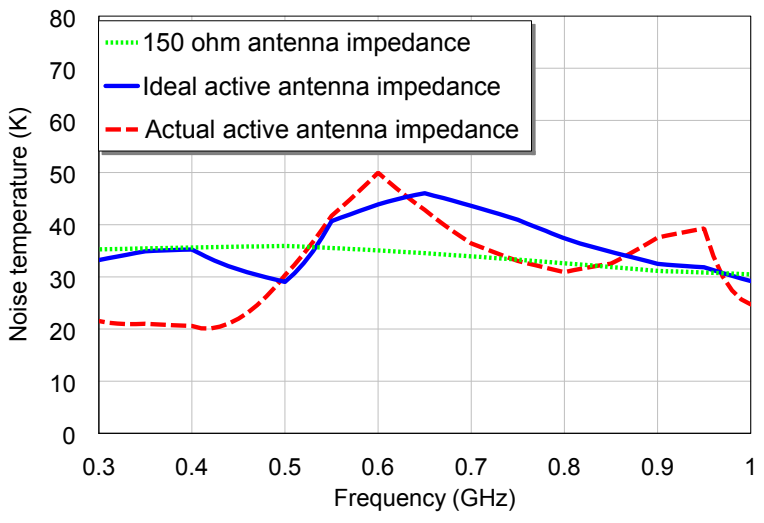

Fig. 14: Simulated noise temperature for different source conditions: $150 \Omega$, active antenna impedance with ideal loading conditions, and actual active antenna impedance.

\section{Conclusions}

This paper describes the design and characterization process of an active receiving array, developed for the mid-frequency range (from $300 \mathrm{MHz}$ to $1000 \mathrm{MHz}$ ) of the SKA. One of the main advantages provided by the proposed solution is the use of differential amplifiers directly connected to the bunny-ear antenna elements, in order to reduce the losses in the antenna. This type of differential structures may exhibit scan anomalies, which have been properly analyzed. A novel method based on the dissipation of the odd-mode currents in the antennas, which cause the scan anomaly problem, has been proposed in order to eliminate such anomalies. With respect to the amplifiers, a design based on inductively degenerated transistors has been presented, in order to simultaneously obtain good noise and matching performance. Additionally, the mismatching effects between the antenna and the amplifiers have been analyzed. Finally, a 64-element dual-polarized array has been manufactured and characterized. This radiating structure is able to scan up to $\pm 45 \mathrm{deg}$ while maintaining good reflection parameters. The noise temperature of the system is estimated to be around $50 \mathrm{~K}$. The future lines include the integration of the system (antennas, LNAs and feeding network) and the noise characterization of the complete active array.

Acknowledgements. This contribution has been partially funded by the Marie Curie MCCT-SKADS programme of the European Commission. The authors want to thank to The Netherlands Institute for Radio Astronomy (ASTRON) for their contribution during the design and characterization process of the differential amplifiers.

\section{References}

van Ardenne, A. et al., "The aperture array approach for the Square Kilometre Array”, White paper, May 2003.

Belostotski, L., Haslett, J. W., "Noise figure optimization of inductively degenerated CMOS LNAs with integrated gate inductors", IEEE TCS, 53(7), Jul. 2006.

Dewdney, P.E., Hall, P.J., Schilizzi, R.T., Lazio, T.J.W., "The Square Kilometre Array," Proceedings of the IEEE, Vol. 97, No. 8, Aug. 2009 pp. 1482-1496, ISSN: 0018-9219

Garcia-Perez, O., Garcia-Muñoz, L. E., Gonzalez-Posadas, V., Segovia-Vargas, D., "Source-pull characterization of differential active antennas for radio-astronomy applications", EuWiT 2009, Sep. 2009.

Lera-Acedo, E., Garcia-Muñoz, L.E., Gonzalez-Posadas, V. Vazquez-Roy, J.L., Maaskant, R., Segovia-Vargas, D., "Study and design of a differentially fed tapered slot antenna array", IEEE TAP, 58(1), Jan. 2010.

Morawietz, J., Witvers, R.H., bij de Vaate, J.G., Woestenburg, E.E.M., "Noise characterization of ultra low noise differential amplifiers for next generation radiotelescopes", EuMC 2007, Oct. 2007.

Pozar, D.M., "The active element pattern", IEEE TAP, 42(8), Aug. 2009. 